\title{
An Extension of the Theory of the Chemostat with Feedback of Organisms. Its Experimental Realization with a Yeast Culture
}

\author{
By S. J. PIRT AND W. M. KUROWSKI \\ Department of Microbiology, Queen Elizabeth College, \\ University of London, Campden Hill, London, W.8
}

(Accepted for publication 4 September 1970)

\begin{abstract}
SUMMARY
Homogeneous continuous-flow culture with some mechanical means for concentrating the biomass in the fermenter is termed a chemostat with feedback. The theory of the system has been reformulated and extended to include concentration of the biomass outside or inside the culture vessel by means such as centrifugation, filtration or gravity sedimentation. An important feature of the general system is the provision of two effluents, one of concentrated biomass and the other of diluted biomass. Experimentally, feedback was realized by filtration of one effluent stream. By this means, steady states were obtained over a wide range of flow rates; the values of biomass and growth-limiting substrate were in good agreement with the theory. The maximum biomass output rate of the chemostat was increased fourfold.
\end{abstract}

\section{INTRODUCTION}

The chemostat with feedback was first represented by Herbert (I96I) as shown in Fig. I $a$. The continuous culture was depicted with a single effluent stream provided with a device, such as a centrifuge, to concentrate the organisms and return part of the concentrate to the culture. Increase in the organism concentration in the fermenter enables the process rates to be increased above those possible in the simple chemostat (Herbert, Elsworth \& Telling, 1956). This method of increasing microbial process rates is required where the concentration of substrate is fixed and cannot be increased as, for example, in effluent disposal or where the substrate solubility, or toxicity of products limits the possible substrate concentration.

The initial formulation of the theory by Herbert (196I) has been followed in subsequent treatments (for example: Fencl, 1966; Pirt, 1969). Hitherto there have been no reports of experiments deliberately designed to test the theory and to explore the extent to which it can be realized in practice. Indeed, it has seemed too difficult to achieve on the normal laboratory scale because of the lack of a simple device to control the concentration of the biomass and feed a known fraction of it back into the culture, especially under aseptic conditions. Now the re-examination of the system reported here has lead to an extension of the theory and to the development of a simple experimental means of realizing the system. 
THEOR Y

The chemostat with feedback, as originally represented (Herbert, 196I) with a single effluent stream, is shown in Fig. I $a$. In practice it is difficult to conceive of any controllable device for concentrating just the amount of biomass required for the feedback. In general, both concentrated and dilute biomass streams would be generated as shown in Fig. I $b$ and a part of the concentrated stream would be fed back.

Two other systems of 'feed back' are represented in Fig. I. In the system shown in Fig. I $c$ a filter is used to remove from the culture a dilute suspension of organisms; in the extreme case this may be a cell-free fluid. Culture containing concentrated suspension of organisms is taken directly from the culture vessel through a constantlevel device. A fourth system of feedback is depicted in Fig. $\mathrm{I} d$. This system consists of a culture in which the organisms growing at the bottom of the vessel are continuously enriched with biomass sedimenting from the upper zones. Thus effluent drawn from the top of the culture forms the dilute stream of organisms. The concentrated stream is pumped out of the lower part of the culture.

\section{Notation}

$V=$ volume of growing culture in the fermenter (1.)

$F=$ rate of flow of medium into the culture (1./h.)

$D=F / V=$ dilution rate $\left(\mathrm{h}^{-1}\right)$

$s_{\mathrm{r}}=$ concentration of growth-limiting substrate in fresh medium (g./l.)

$s=$ concentration of growth-limiting substrate in culture (g./1.)

$x=$ biomass (organism) concentration (g./l.)

$c=$ fraction of total effluent stream which contains concentrated biomass

$Y=$ growth yield (g. biomass/g. growth-limiting substrate utilized)

$\mu=$ specific growth rate $\left(\mathrm{h} \cdot \cdot^{-1}\right)$

$\mu_{\mathrm{m}}=$ maximum specific growth rate $\left(\mathrm{h} .^{-1}\right)$

$t=$ time (h.)

$a, g, k$ are dimensionless coefficients

$K_{\mathrm{s}}=$ saturation constant (g./1.) for the growth-limiting substrate, in the Monod relation, $\mu=\mu_{\mathrm{m}} s /\left(K_{\mathrm{g}}+s\right)$.

A bar over a symbol (for example, $\bar{x}$ ) indicates that it is a steady-state value.

\section{Chemostat with feedback}

The system represented in Fig. $\mathrm{I} b$ will be considered first. The net rate of medium flow through the fermenter is $F$. A fraction $a$ of the total flow out of the fermenter $\left(F_{\mathrm{s}}\right)$ is fed back; thus $F_{\mathrm{s}}=\mathrm{F}+a F_{\mathrm{s}}$, whence $F_{\mathrm{s}}=F /(\mathrm{I}-a)$. It is supposed that sedimentation of organisms concentrates the biomass by the factor $g$ so that the concentration of biomass in the feedback line is $g x$ where $g>\mathrm{r}$. Only a part of the biomass concentrate is fed back, the remainder forms the concentrated biomass effluent with flow rate $c F$. The dilute biomass effluent has the concentration $k x$ where $k<\mathrm{I}$. The subsequent analysis is made with the simplifying assumption that the time spent by the culture in the feedback line is negligible.

The rate of biomass production can be obtained from the organism balance for the fermenter, which is

$$
\text { increase in biomass }=\text { growth }- \text { output }+ \text { feedback. }
$$


In the infinitely small time interval $\mathrm{d} t$ the balance will be

$$
V \mathrm{~d} x=\mu x V \mathrm{~d} t-F_{\mathrm{s}} x \mathrm{~d} t+a F_{\mathrm{s}} g x \mathrm{~d} t .
$$

On dividing through by $\mathrm{d} t$, substituting for $F_{\mathrm{s}}$ and putting $D=F / V$ we obtain

Let $\beta=(\mathrm{I}-a g) /(\mathrm{I}-a)$ then

$$
\frac{\mathrm{d} x}{\mathrm{~d} t}=\left\{\mu-\frac{(\mathrm{I}-a g)}{\mathrm{I}-a} D\right\} x .
$$

$$
\frac{\mathrm{d} x}{\mathrm{~d} t}=(\mu-\beta D) x .
$$

In the steady state $\mathrm{d} x / \mathrm{d} t=0$ and $\bar{\mu}=\beta D$. It should be noted that $\beta$ must be a positive fraction since $a g<\mathrm{I}$ (or the biomass fed back would not be $<100 \%$, which is essential for steady states) and $a g>a$.

The balance for the growth-limiting substrate in the fermenter is given by

Increase in substrate $=$ input - substrate used for growth - output + feedback, that is,

$$
V \mathrm{~d} s=F s_{\mathrm{r}} \mathrm{d} t-(\mu x / Y) V \mathrm{~d} t-F_{\mathrm{g}} s \mathrm{~d} t+a F_{\mathrm{g}} s \mathrm{~d} t
$$

(a)

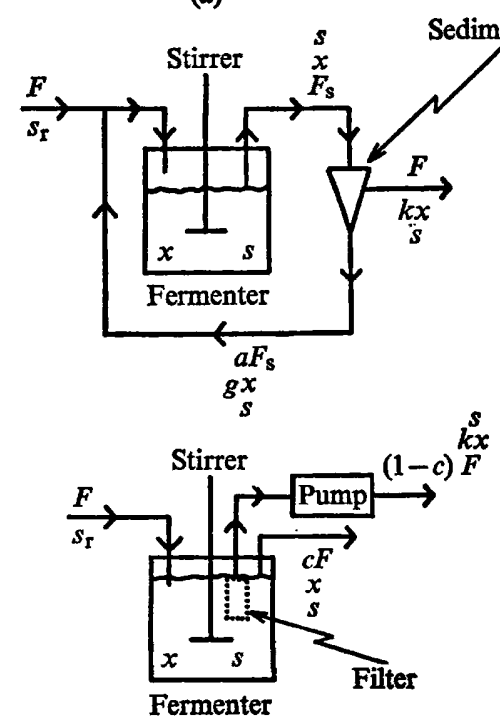

(c) (b)

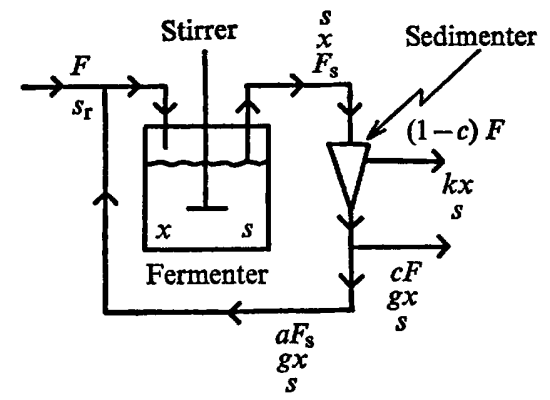

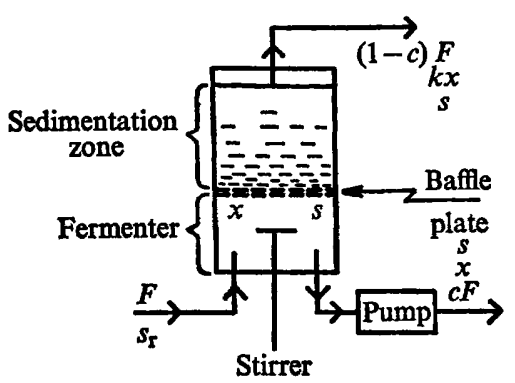

(d)

Fig. I. The chemostat with feedback of biomass. The symbols represent the concentrations of biomass $(x)$, growth-limiting substrate $(s)$ and flow rate $(F)$ at various points. $(a)$ Single effluent system with sedimenter which concentrates the biomass by the factor $g$ in the feedback stream. (b) Dual effluent system with concentrated biomass effluent (flow rate, $c F$ ) and diluted biomass (flow rate, $(\mathrm{I}-c) F)$. (c) Filter feedback system. The diluted biomass effluent is extracted through the filter. $(d)$ Fermenter with sedimentation zone. The baffle plate would serve to separate the homogeneous fermenter zone from the sedimentation zone. Concentrated biomass is removed at the base of the fermenter and diluted biomass from the top of the sedimenter. 
On dividing by $V \mathrm{~d} t$ we obtain

$$
\frac{\mathrm{d} s}{\mathrm{~d} t}=D\left(s_{\mathrm{r}}-s\right)-\frac{\mu x}{Y}
$$

To solve for the steady-state values of $x$ and $s$, which are obtained when $\mathrm{d} x / \mathrm{d} t=\mathrm{d} s / \mathrm{d} t=0$, we substitute for the variable $\mu$ by means of the Monod relation, $\mu=\mu_{\mathrm{m}} s /\left(K_{\mathrm{s}}+s\right)$. Hence

$$
\bar{s}=\frac{\beta D K_{\mathrm{s}}}{\mu_{\mathrm{m}}-\beta D} \quad \text { and } \bar{x}=\frac{Y}{\beta}\left(s_{\mathrm{r}}-\bar{s}\right) .
$$

At the critical dilution rate $\left(D_{\mathrm{c}}\right)$ the organisms grow at the maximum rate which the medium will allow. This value of the dilution rate is obtained by putting $\mu=\beta D_{\mathrm{c}}$ and $s=s_{\mathrm{r}}$ in the Monod relation, that is,

and when $K_{\mathrm{s}} \ll s_{\mathrm{r}}, D_{\mathrm{c}} \approx \mu_{\mathrm{m}} / \beta$.

$$
D_{\mathrm{c}}=\frac{\mu_{\mathrm{m}}}{\beta} \frac{s_{\mathrm{r}}}{K_{\mathrm{s}}+s_{\mathrm{r}}}
$$

The concentration of biomass in the dilute effluent $(k x)$ can be found from the biomass balance

biomass leaving fermenter $=$ biomass in concentrated effluent

$$
\text { + biomass in diluted effluent + biomass in feedback, }
$$

that is,

$$
F_{\mathrm{g}} x=c F g x+(\mathrm{I}-c) F k x+a F_{\mathrm{s}} g x .
$$

On simplifying and rearranging we obtain

$$
k=\frac{\beta-c g}{\mathrm{I}-c} .
$$

The single effluent system shown in Fig. I $a$ can be regarded as a special case of the dual effluent system in which $c=0$. Hence the equations for $\bar{x}$ and $\bar{s}$ are unaffected but $k=\beta$.

\section{Chemostat with filter feedback}

The filter feedback system is depicted in Fig. I $c$. Just as in the preceding system there are two effluent streams, one containing concentrated biomass and the other diluted biomass. The biomass balance for the fermenter is

increase in biomass $=$ growth - output in concentrated stream

that is, for the infinitely small time interval $\mathrm{d} t$

$$
\text { - output in diluted stream, }
$$

$$
V \mathrm{~d} x=\mu x V \mathrm{~d} t-c F x \mathrm{~d} t-(\mathrm{I}-c) F k x \mathrm{~d} t .
$$

On substituting $D=F / V$ and rearranging we obtain

$$
\frac{\mathrm{d} x}{\mathrm{~d} t}=[\mu-\{c+(\mathrm{I}-c) k\} D] x \text {. }
$$

Let $\gamma=c+(\mathrm{I}-c) k$, then the value of $\mu$ in the steady state, when $\mathrm{d} x / \mathrm{d} t=0$, is $\bar{\mu}=\gamma D$. 
The balance for growth-limiting substrate is

increase in substrate $=$ input - substrate used for growth that is,

hence

$$
V \mathrm{~d} s=F s_{\mathrm{r}} \mathrm{d} t-(\mu x / Y) V \mathrm{~d} t-F s \mathrm{~d} t
$$

$$
\frac{\mathrm{d} s}{\mathrm{~d} t}=D\left(s_{\mathrm{r}}-s\right)-\frac{\mu x}{Y} .
$$

Putting $\mathrm{d} x / \mathrm{d} t=0$ and $\mathrm{d} s / \mathrm{d} t=0$ in equations (II) and (I3) and substituting for $\mu$ we obtain for the steady state

$$
\bar{s}=\frac{\gamma D K_{\mathrm{s}}}{\mu_{\mathrm{m}}-\gamma D} \quad \text { and } \bar{x}=\frac{Y}{\gamma}\left(s_{\mathrm{r}}-\bar{s}\right)
$$

The critical dilution rate is given by substituting $\mu=\gamma D_{\mathrm{c}}$ and $s=s_{\mathrm{r}}$ in the Monod relation; hence $D_{\mathrm{c}} \approx \mu_{\mathrm{m}} / \gamma$ when $s_{\mathrm{r}} \gg K_{\mathrm{a}}$.

An important special case of the chemostat with filter feedback occurs when cellfree medium is extracted through the filter. Then $k=0$ and $\gamma=c$, and the steadystate values of $x$ and $s$ become

and

$$
\bar{s}=\frac{c D K_{s}}{\mu_{\mathrm{m}}-c D}
$$

$$
\bar{x}=\frac{Y}{c}\left(s_{\mathrm{r}}-\bar{s}\right)
$$

The fourth system of feedback depicted in Fig. I $d$ theoretically is identical with the filter feedback system. This involves the simplifying assumptions that all the growth occurs in the lower zone (to which nutrient is added) and that the organisms are unaffected by their stay in the sedimentation zone.

In the three cases of feedback considered the expressions giving the steady-state concentrations of organisms and growth-limiting substrate are formally the same, the only difference being in the factor $\beta, \gamma$ or $c$, the reciprocal of which expresses the increase in the biomass concentration over that which would be obtained in the chemostat without feedback. Because of its fundamental significance, the factor $\mathrm{I} / \beta, \mathrm{I} / \gamma$ or $\mathrm{I} / \mathrm{c}$ is termed the 'feedback factor'. The theory has been tested by use of the filter feedback system.

\section{METHODS}

Apparatus. The continuous-flow cultures were made in the CECA (Gallenkamp, Christopher Street, London, E.C. 2) type chemostat. The culture vessel was based on the $152 \times 305 \mathrm{~mm}$. Pyrex pipeline as used by Elsworth, Meakin, Pirt \& Capell (1956). The culture volume was in the range $\mathrm{I} \cdot 5$ to $2 \cdot 01$.

Culture filtration. The filter feedback system was achieved by insertion of Whatman glass fibre filter tubes (Reeve Angel Ltd, I4 New Bridge Street, London, E.C. 4) in the culture vessel. The filters excluded particles $>2 \mu \mathrm{m}$. diameter. For filtration with a yeast concentration up to I. $5 \mathrm{~g}$. dry wt./l., three filters of type A I $(60 \times$ I $2 \mathrm{~mm}$., total filter area $\left.78 \mathrm{~cm} .^{2}\right)$ were used; for filtration rates with yeast cell densities up to $3.5 \mathrm{~g}$. dry wt./1. four filters of type B $2\left(60 \times 26 \mathrm{~mm}\right.$.; total filter area $\left.180 \mathrm{~cm} .^{2}\right)$ were inserted 
in the fermenter. One end of each filter was closed with a rubber bung; the other end was connected by means of a polypropylene adaptor (Portex Ltd, Hythe, Kent) to a stainless steel tube and the joints sealed with Araldite. The stainless steel tube was passed through a bung in one of the ports on the lid of the fermenter. The steel tube was then connected by silicone tubing to one of the peristaltic pumps on the apparatus which provided the suction for filtration. The filtration rate (up to $2.51 . / \mathrm{h}$.) was monitored by one of the burettes provided for medium flow rate measurement.

Culture conditions. The culture temperature was controlled at $30^{\circ}$. During the steady states the $\mathrm{pH}$ value was maintained at $5.5 \pm 0 . \mathrm{I}$ by the buffer in the medium. Antifoam (0.I ml. polypropylene glycol 2000) was added every $2 \mathrm{~h}$. Normally, vortex aeration by free agitation was used in the vessel. However, because the presence of the filters partially interfered with the vortex formation, it was decided to use a relatively low stirrer speed (900 rev./min.) with air sparged through the culture at $600 \mathrm{ml} / \mathrm{min}$. The sparger consisted of a steel tube of bore $2 \mathrm{~mm}$. dipping to the bottom of the culture vessel.

Medium. The nutrient medium had the following composition (g./l.): glucose, I.5; $\mathrm{Na}_{2} \mathrm{HPO}_{4} .2 \mathrm{H}_{2} \mathrm{O}, \mathrm{I} \cdot 75 ; \mathrm{KH}_{2} \mathrm{PO}_{4}, 13.5 ; \mathrm{MgSO}_{4} .7 \mathrm{H}_{2} \mathrm{O}, 0.25 ; \mathrm{CaCl}_{2} .2 \mathrm{H}_{2} \mathrm{O}, 0.05$; $\mathrm{NH}_{4} \mathrm{Cl}, 2 \cdot 0 ; \mathrm{FeSO}_{4} \cdot 7 \mathrm{H}_{2} \mathrm{O}, 3 \times 10^{-6} ; \mathrm{KMnO}_{4}, 3 \times 10^{-7} ; \mathrm{ZnSO}_{4} \cdot 7 \mathrm{H}_{2} \mathrm{O}, 4.2 \times 10^{-6}$; $\mathrm{CoNO}_{3} .6 \mathrm{H}_{2} \mathrm{O}, 5 \times \mathrm{IO}^{-7} ; \mathrm{KI}, \mathrm{I} \cdot 0 \times \mathrm{I}^{-6}$; m-inositol, $\mathrm{I} \cdot 25 \times \mathrm{IO}^{-3}$; thiamine, $2.5 \times \mathrm{IO}^{-4}$; nicotinic acid, $2.5 \times 10^{-4}$; riboflavin, $2.5 \times 10^{-4}$; calcium pantothenate, $2.5 \times 10^{-4}$; $p$-aminobenzoic acid, $\mathrm{I} \cdot 25 \times \mathrm{IO}^{-4}$; pyridoxin, $2.5 \times 10^{-4} ; d$-biotin, $6.25 \times 10^{-6}$.

The medium was prepared in four parts: (I) glucose solution, (2) phosphates, (3) ammonium chloride and magnesium sulphate, (4) the calcium chloride, vitamins and other minerals. Solutions (I), (2) and (3) were sterilized by autoclaving at $12 \mathrm{I}^{\circ}$; solution (4) was sterilized by Seitz filtration. To make 201 . of medium, 10 1. solution (I), 6 l. solution (2), 3.945 1. solution (3) and 0.055 1. solution (4) were mixed aseptically.

Inoculum. A loopful of the organism (Saccharomyces cerevisiae, National Collection of Yeast Cultures, strain 229) was transferred to $5 \mathrm{ml}$. of the nutrient medium and incubated at $30^{\circ}$ until turbid. This culture was used to inoculate $200 \mathrm{ml}$. nutrient medium in a 21 . flask. After overnight incubation on an orbital shaker at $30^{\circ}$ the culture was used to inoculate the CECA fermenter.

Analytical methods. The turbidity of the organism suspension was read on an EEL colorimeter with a green filter. The turbidity was related to the mass of the waterwashed organism dried at $105^{\circ}$. Residual glucose in the culture was determined with glucose oxidase after rapid removal of organisms by membrane filtration.

\section{RESULTS}

The experiments were designed to test the theoretical equations relating the steadystate concentrations of biomass and growth-limiting substrate to the dilution rate and specific growth rate. Consequently it was necessary to vary the dilution rate, the feedback factor $(\mathrm{I} / c)$ and determine the steady-state concentrations of yeast and of glucose (the growth-limiting substrate). A yeast rather than a bacterial species was used as the test organism because filtration of the culture was thereby greatly facilitated. Also, because of the low $\mathrm{pH}$ value, the culture was unlikely to support growth of any bacterial contaminant. This 'resistance' was useful because, during cultivation, filters were changed by removing the top plate of the fermenter. During the latter stages of 
the work there was a low degree of bacterial contamination $(<3$ bacteria/Ioo yeast cells). This low degree of bacterial contamination contributed a negligible amount to the biomass and, it was shown, had no effect on the biomass:turbidity ratio.

The filtered effluent stream was virtually cell-free; the ratio of the number of yeasts in the filtrate to the number in the culture being about $10^{-4}$. The useful life of the filters varied greatly with the filtration rate and the yeast concentration. When the filters started to block during steady-state operation, the filtration rate was increased by periodic reversal of the flow through the filter so as to pump back about $15 \mathrm{ml}$. of filtrate. This could conveniently be done since the flow inducer on the CECA was provided with a switch to reverse the direction of flow. The complete procedure of backflushing consisted of pumping $15 \mathrm{ml}$. of filtrate into the burette used for flow-rate measurement, then pumping this filtrate back through the filters. The time for this operation was from 30 to $60 \mathrm{sec}$. The frequency of back-flushing increased with the filtration rate and yeast concentration. The frequency was less than once every $10 \mathrm{~min}$. except at the highest dilution rate (about $\mathrm{I} \cdot 5 \mathrm{~h}^{-1}$ ) when filters were back-flushed at $4 \mathrm{~min}$. intervals. During this procedure the medium supply was stopped. This necessitated a correction to the medium flow rate, which was taken to be the mean rate given by, pumping rate $x$ (sum of 'on' periods/sum of 'on' and 'off' periods).

\section{Determination of critical dilution rate $\left(D_{\mathrm{c}}\right)$}

The critical dilution rate was determined from the rate of wash-out of the culture when the dilution rate exceeded $D_{\mathrm{c}}$. We then have, when $s \gg K_{\mathrm{s}}, \mu \approx \mu_{\mathrm{m}}$ and $\mu_{\mathrm{m}}$ is given by

$$
\ln x=\left(\mu_{\mathrm{m}}-c D\right) t+\ln x_{0}
$$

where $x_{0}=$ biomass when $t=0$. Thus $\mu_{\mathrm{m}}$ can be calculated from the slope of a plot of $\ln x$ against $t$ and $D_{\mathrm{c}}$ was given by $\mu_{\mathrm{m}} / c$. In order to facilitate the filtration and maintain $c$ constant at the high values of $D$ used for wash-out (I.24 h..$^{-1}$ with $c=0.47$ and $\mathrm{I} \cdot 83 \mathrm{~h}^{-1}$ with $c=0 \cdot 25$ ), the yeast concentration was first reduced, by wash-out, to about a quarter of the maximum value. Then excellent straight-line relations were found between $\ln x$ and $t$ over a period of $1.5 \mathrm{~h}$. or more. The value for $\mu_{\mathrm{m}}$ found was $0.42 \pm 0.0 \mathrm{I} \mathrm{h} .^{-1}$, with $c=0,0.47$ and 0.25 . The value of $\mu_{\mathrm{m}}$ found in batch culture was virtually identical $\left(0.4 \mathrm{I} \mathrm{h} .^{-1}\right)$. Thus the critical dilution rates were equal to the theoretical values.

Steady states. The criterion for the steady state was that three turbidity measurements taken at $30 \mathrm{~min}$. intervals agreed within $1.5 \%$ and showed no upward or downward trend. The steady-state values of the yeast and glucose concentrations are shown in Fig. 2 and 3. The glucose concentrations with the lower dilution rates and higher feedback factor were too low to be measured $(<5 \mu \mathrm{g} . / \mathrm{ml}$.). The value of the saturation $\left(K_{\mathrm{a}}\right)$ was found to be $0.025 \mathrm{~g}$./1. from a plot of the reciprocals of $\bar{\mu}$ and $\vec{s}$ (Fig. 4).

The graphs show good agreement between the experimental and theoretical values of $\bar{x}$ and $\bar{s}$. In the case of the chemostat without feedback, at the high dilution rates $\bar{x}$ rose above the theoretical value. This deviation can be attributed to some growth of the yeast on the instrument probes in the culture vessel, producing an 'apparatus effect' (Herbert, et al. 1956). With $c=0.25$, near to the critical dilution rate the biomass deviated below the theoretical value. This deviation could be due to error in the 


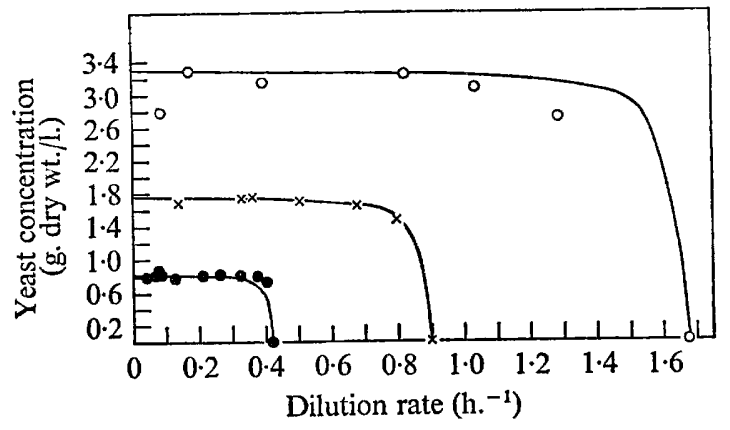

Fig. 2

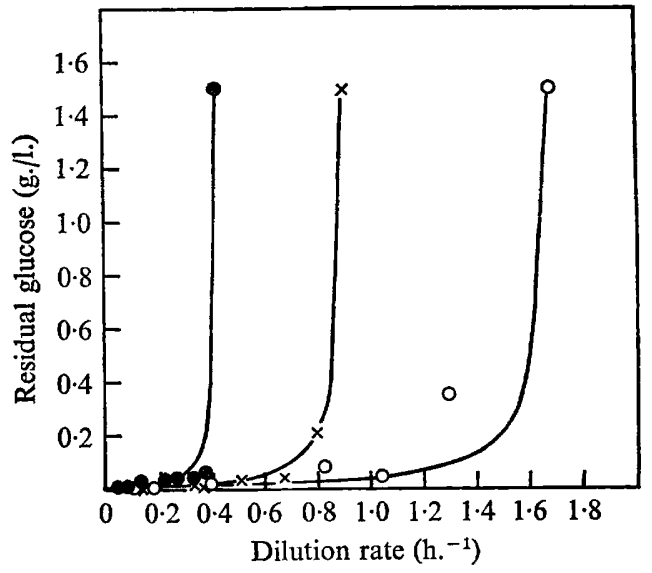

Fig. 3

Fig. 2. Yeast concentration as function of dilution rate: $\bullet$, without feedback; $\times$, with feedback $(\mathrm{I} / c=2 \cdot \mathrm{I} 4) ; O$, with feedback $(\mathrm{I} / c=4 \cdot 0)$. The lines drawn are the theoretical curves with $Y=0.55 \mathrm{~g}$. yeast dry wt. $/ \mathrm{g}$. glucose, $K_{\mathrm{s}}=0.025 \mathrm{~g} . / \mathrm{l} ., s_{\mathrm{r}}=\mathrm{I} \cdot 50 \mathrm{~g} . / \mathrm{l}$., $\mu_{\mathrm{m}}=0.42 \mathrm{~h}^{-1}$.

Fig. 3. Residual glucose concentrations in steady states at different dilution rates: without feedback; $X$, with feedback $(\mathrm{I} / c=2 \cdot \mathrm{I} 4) ; O$, with feedback $(\mathrm{I} / c=4 \cdot 0)$.

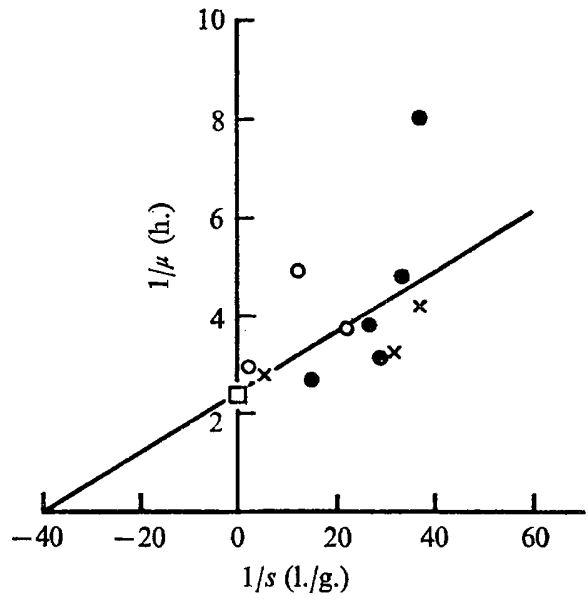

Fig. 4

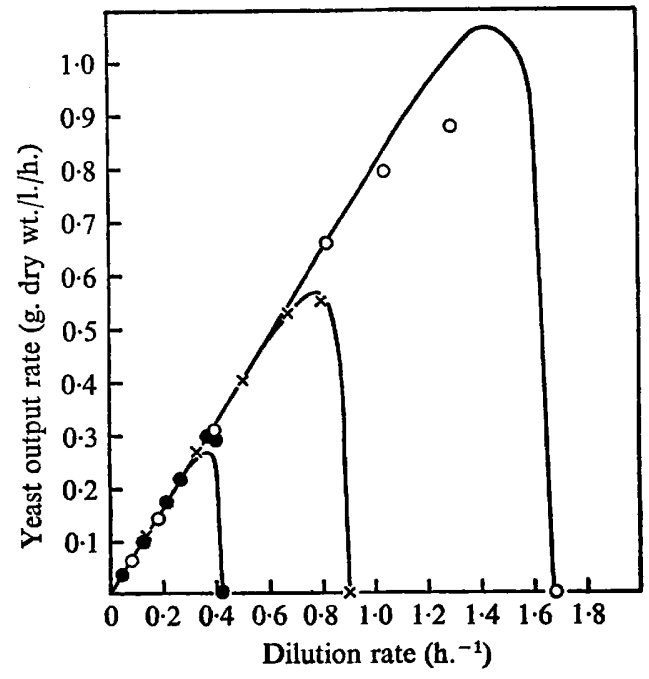

Fig. 5

Fig. 4. Plot of reciprocal of growth-limiting substrate concentration ( $1 / s)$ against the reciprocal of the specific growth rate $(\mathrm{I} / \mu): 0$, no feedback; $\times$, with feedback $(1 / c=2 \cdot 14)$; $O$, with feedback $(\mathrm{I} / c=4 \cdot 0) ; \square, \mu_{\mathrm{m}}$ from unsteady state.

Fig. 5. Output rate of biomass $(D \bar{x})$ in steady states at different dilution rates: 0 , without feedback; $X$, with feedback $(1 / c=2 \cdot 14) ; O$, with feedback $(1 / c=4 \cdot 0)$. The lines drawn are the theoretical curves with $Y=0.55 \mathrm{~g}$. yeast dry wt. $/ 1 ., K_{\mathrm{g}}=0.025 \mathrm{~g} . / \mathrm{l}$., $s_{\mathrm{r}}=\mathrm{I} \cdot 50 \mathrm{~g} . / 1$., $\mu_{\mathrm{m}}=0.42 \mathrm{~h}^{-1}$. 
flow rate introduced by the frequent back-flushing of the filters necessary at such a high flow rate, combined with high yeast concentration. At this point the filtration technique was stretched to its limit. The deviation in $\bar{x}$ at the lowest dilution rates can be attributed to maintenance energy effects (Pirt, 1965) which were not allowed for.

The rates of biomass output are plotted in Fig. 5. They show how dramatically feedback can increase the output rate of a chemostat.

\section{DISCUSSION}

The theory of the chemostat with feedback formulated here unifies various systems of continuous culture aimed at increasing the concentration of biomass in a culture with a fixed substrate concentration. This new analysis, in contrast to the original formulation (Herbert, 196I), shows that in general the system requires two effluent streams, one of concentrated, and the other of diluted biomass. The results show that filtration of part of the effluent stream provides a simple means of realizing feedback on the laboratory scale. Sortland \& Wilke (I969) devised a 'filtration fermenter' from which cell-free medium was extracted through a rotating membrane filter whilst concentrated biomass was removed in a second stream. However, the theory of this system was not related to that of the chemostat with feedback and the experimental data dealt with only transient states and did not indicate the range of steady states possible.

The 'tower fermenter' (Royston, I966), which is used in the beer fermentation, is a system which achieves feedback by sedimentation in the fermenter but the fermenter has only one outlet provided at the top of the fermenter from which a diluted biomass stream emerges. In such a culture the biomass concentration will continually increase until the culture becomes solid and therefore only unsteady states can be obtained. With this type of fermenter it seems to be the practice to run off periodically some of the dense culture at the base. If the tower fermenter were provided with an outlet for the concentrated biomass at the base, it would represent a feedback system of the type shown in Fig. I $d$. The theory is, of course, oversimplified in that it assumes the organisms would not change in the sedimentation zone. Clearly, if they remained long in the sedimentation zone the organisms would go into a resting state or stationary phase of growth which would take some time to reverse when the organisms re-entered the fermenter zone.

The laboratory realization of the chemostat with feedback makes possible many studies on the acceleration of microbial processes such as brewing, effluent disposal, utilization of wastes such as whey and mineral leaching, where normally the substrate concentration is fixed and consequently the organism concentration is low without feedback. Feedback also provides a means of increasing output rate where an inhibitory product is formed at a concentration $(P)$ proportional to the substrate concentration, that is, $P=b s_{\mathrm{r}}$ where $b$ is a constant. Such a process could be oxidation of ammonia to nitrite by Nitrosomonas. The method is also one sought after in animal tissue cell culture (for example, Himmelfarb, Thayer \& Martin, 1969) where either nutrient solubility, in particular, cystine, or an unknown growth-limiting factor limits maximum cell concentration.

The filtration technique needs to be developed. The filters used in this study are probably much more limited in their applicability to bacterial cultures. New types of filter such as those of sintered steel (Pall (U.K.) Ltd, Walton Road, Farlington, Portsmouth, Hants) may provide the desired improvement in performance. 


\section{REFERENCES}

Elsworth, R., Meakin, L. R. P., Pirt, S. J. \& Capell, G. H. (1956). A two litre scale continuous culture apparatus for micro-organisms. Journal of Applied Bacteriology 19, 264-278.

FenCL, Z. (1966). A theoretical analysis of continuous culture systems. In Theoretical and Methodological Basis of Continuous Culture of Micro-organisms. Edited by I. Malek \& Z. Fencl. New York: Academic Press.

Herbert, D. (196I). A theoretical analysis of continuous culture systems. In Continuous Culture, monograph no. 12, pp. 2I-53. London: Society of Chemistry and Industry.

Herbert, D., Elsworth, R. \& Telling, R. C. (I956). The continuous culture of bacteria: a theoretical and experimental study. Journal of General Microbiology 14, 60I-622.

Himmelfarb, P., Thayer, P. S. \& MARTin, M. E. (1969). Spin filter culture: the propagation of mammalian cells in suspension. Science, New York 164, 555-557.

PIRT, S. J. (1965). The maintenance energy of bacteria in growing cultures. Proceedings of the Royal Society B 163, 224-23I.

PIRT, S. J. (1969). Microbial growth and product formation. Symposia of the Society for General Microbiology 19, 199-221.

Royston, M. G. (1966). Tower fermentation of beer. Process Biochemistry 1, 215-221.

Sortland, L. D. \& Wilke, C. R. (I969). Growth of Streptococcus faecalis in dense culture. Biotechnology and Bioengineering II, 805-841. 